\title{
Screening for sarcopenia and sarcopenic obesity in community-dwelling older adults
}

\author{
J. Jones, F. Lemmon, I. Arvanitidou, E. Bannerman, I. Davidson and L. Wyness \\ Queen Margaret University, Edinburgh EH21 6UU, UK
}

It is well established that sarcopenia and sarcopenic obesity are geriatric syndromes which are common within the $\mathrm{UK}^{(1)}$ and can be prevented or their progression delayed through targeted nutrition and lifestyle advice ${ }^{(2)}$. Screening tools are commonly used in the UK to identify undernutrition and the most commonly used nutrition screening tool in the UK is the Malnutrition Universal Screening Tool 'MUST'(3). This tool is recognised as a robust validated tool to identify undernutrition however its ability to detect sarcopenia and sarcopenic obesity has not been established. The aims of this study were to 1) investigate the prevalence of malnutrition, sarcopenia and sarcopenic obesity in community-dwelling older adults and 2) explore the suitability of 'MUST' as a screening tool for sarcopenia and sarcopenic obesity.

Community-dwelling older adults (aged 65 years and over) were recruited from a range of social groups and lunch clubs across central Scotland. Participants were screened for undernutrition using 'MUST'(3) and for sarcopenia using the European consensus statements criteria ${ }^{(4)}$

Fifty nine older adults $(21 \mathrm{~m}, 38 \mathrm{f})$ mean(sd) age $81 \cdot 1(7 \cdot 2)$ years with a mean(sd) BMI of $27 \cdot 4(4 \cdot 3) \mathrm{kg} / \mathrm{m}^{2}$. Participants were found to be of relatively poor functional status with a mean(sd) gait speed of $0 \cdot 69(0 \cdot 38) \mathrm{m} / \mathrm{s}$ and a mean(sd) handgrip strength of $16 \cdot 8(7 \cdot 8) \mathrm{kg}$. Low levels of nutritional risk and high levels of sarcopenia and sarcopenic obesity were found.

\begin{tabular}{lllll}
\hline & $\mathrm{n}$ & Nutritional risk & No sarcopenia & Sarcopenia \\
\hline Men & 21 & 2 & 6 & 4 \\
Women & 38 & 1 & 26 & 2 \\
Total & $\mathbf{5 9}$ & $\mathbf{3}(\mathbf{5} \%)$ & $\mathbf{3 2}(\mathbf{5 4} \%)$ & 11 \\
\hline
\end{tabular}

This study suggests that the prevalence of sarcopenia (with or without obesity) in this specific population is relatively high compared to the prevalence found in a previous UK study carried out in a similar population ${ }^{(1)}$ which estimated the prevalence of sarcopenia in UK community-dwelling older adults to be $4.6 \%$ among men and 7.9\% among women. As 'MUST' was not developed as a measure of sarcopenia it is not surprising that when used on its own it does not identify sarcopenia in community-dwelling older adults. However, as the development or progression of sarcopenia and sarcopenic obesity can be prevented or delayed through dietary and activity based interventions, including a measure of function such as hand grip or gait speed, may be a helpful complement to 'MUST'. This has the potential to enable early identification of a range of nutrition related disorders and thus enable timely targeted advice.

1. Patel HP, Syddall HE, Jameson K et al. (2013) Prevalence of sarcopenia in community-dwelling older people in the UK using the European Working Group on Sarcopenia in Older People (EWGSOP) definition: findings from the Hertfordshire Cohort Study (HCS) Age and Ageing 42, 378-84.

2. Rom O, Kaisari S, Aizenbud D et al. Lifestyle and Sarcopenia-Etiology, Prevention, and Treatment. RMMJ 2012; 3 :e0024. doi:10.5041/ RMMJ.10091

3. BAPEN (British Association for Parenteral and Enteral Nutrition) (2003) Malnutrition Universal Screening Tool ('MUST'). London: BAPEN

4. Cruz-Jentoft AJ, Baeyens JP, Bauer JM et al. (2010) Sarcopenia: European consensus on definition and diagnosis: report of the European working group on sarcopenia in older people. Age and Ageing, 39, 412-423. 